Original Article

\title{
Online Hemodiafiltration Versus High-Flux Hemodialysis in Pediatric Patients.
}

\author{
Rasha Essam Eldin Galal', Dina Hesham² \\ 1- Department of Pediatrics, Cairo University \\ 2- Department of Chemical Pathology, Cairo University
}

\begin{abstract}
Introduction Online haemodiafiltration (OL-HDF is associated with increased convective clearance compared to HD, even with a high flux membrane. The aim of this study was to compare the effect of OL-HDF and high-flux HD (HF-HD) on anemia, metabolic bone disease markers and dialysis efficiency.
\end{abstract}

\section{Methods}

In this prospective study, 16 children, on hemodialysis for at least three months, were shifted from HF-HD to OL-HDF for a period of 3 months on each modality. The mean age of patients was $10.31 \pm 3.28$ years, and the mean duration on HD was $57.50 \pm 38.24$ months. Blood pressure, hemoglobin $(\mathrm{Hb})$, Calcium, phosphorus and Parathyroid hormone were measured after 3 months of HF-HD and again after 3 months of OL-HDF.

\section{Results}

In our study, dialysis efficiency expressed by Kt/V was significantly improved by OL-HDF (2.07 \pm 0.47$)$ as compared to HFHD (1.75 \pm 0.46$)$; $p$ value of 0.006. Significantly lower levels of Phosphorus and Parathyroid hormone (PTH) on HF-HD compared to OL-HDF were observed $(4.25 \pm 1.3$ to $4.92 \pm 1.9 \mathrm{mg} / \mathrm{dl}$ on OL-HDF, p value 0.04 ; and $404 \pm 476$ to $749 \pm 79 \mathrm{~g} / \mathrm{dl}$, $\mathrm{p}$ value 0.001 , respectively). The levels of $\mathrm{Hb}$, hematocrit (HCT) and ferritin, Erythropoietin dose requirements were not significantly differnet. Systolic blood pressure values showed significant reduction on HF-HD (111.4 \pm 12.3 and $114.4 \pm 10.9$ $\mathrm{mmHg}$ respectively, $\mathrm{p}$ value 0.013 ).

\section{Conclusions}

Over a period of three months, oHDF was not associated with better anemia management and nutrition when compared to HF-HD, while HDF proved to be more beneficial in dialysis efficiency. HF-HD was more effective in controlling BP and metabolic bone disease.

\section{Keywords}

Anemia, dialysis efficiency, high-flux haemodialysis, metabolic bone disease, online haemodiafiltration, outcome, pediatric.

\section{Correspondence}

Rasha E. E. Galal

Department of Pediatrics, Faculty of Medicine, Abu el Rish Children's hospital Cairo University, Cairo, Egypt. Email: raschaessam@yahoo.com

\author{
geget : The Journal of the Egyptian Society of Pediatric Nephrology and Transplantation (ESPNT) \\ geget https://geget.journals.ekb.eg/ \\ Published by ESPNT http://espnt.net/ \\ Cohosted by Egyptian Knowledge Bank https://www.ekb.eg
}




\section{Introduction}

Theoretically, online hemodiafiltration (OL-HDF is associated with increased convective clearance compared to HD, even with a high flux membrane, however clinical results are deficient especially in children. Our goal was to examine and compare the outcomes of both modalities.

HDF is a hybrid therapy combining within the same dialysis module the two main solute transport mechanisms: Diffusion - as in HD - where the rate of diffusion of molecules is inversely proportional to the square root of the molecular weight, in which larger molecules have a relatively low speed of diffusion and therefore a relatively slow clearance by HD, and Convection which depends on the solvent drag where molecules- irrespective of their molecular weight- are transported across the membrane by bulk fluid flow, hence increasing the removal of those middle and large sized molecules that are poorly cleared by diffusion therapies [1]. Dialyzer membrane in HD is one of the main determinants of dialysis effectiveness. HD using high-flux dialyzers has the ability to remove more middle molecular weight uremic toxins such as $\beta 2$-microglobulin than low-flux HD, because of their higher porosity [2].

\section{Patients and Methods}

This study was conducted in the hemodialysis (HD) unit of the Pediatric Nephrology Unit at Abu el Rish Children's hospital, Cairo University during the period from February to August 2010. The study protocol conformed to the ethical guidelines of the 1975 Helsinki declaration and was approved by the local ethical committee of the Pediatric Department, Faculty of Medicine, and Cairo University. Sixteen children 4-16 years of age on regular HD for at least three months were recruited. Inclusion criteria included patients on regular hemodialysis for at least three months prior to the study, while patients who received blood transfusions or were admitted to the hospital in the 30 day period prior to the study, or those with irregular attendance of hemodialysis were excluded. Informed consent was obtained from all participants. All the patients were treated with the dialyzer polysulfone FX-40 or 50 (Fresenius Medical Care, Bad Hamburg, Germany) and Fresenius 4008/5008 dialysis systems (Fresenius Medical Care), receiving either standard high-flux HD and ultrapure bicarbonate-based dialysis fluid or online HDF treatment in postdilution mode and ultra-pure bicarbonate-based dialysis fluid. The substitution flow rate in HDF was set at $20-25 \%$ of the blood flow rate. A control of transmembrane pressure was assessed by the Fresenius 4008/5008 machines. Duration of dialysis session was 4 hours for both standard HD and HDF. Median dialysis vintage was 6.2 $(0.5-35)$ years. The delivered dialysis dose was assessed by calculating the equilibrated urea $\mathrm{Kt} / \mathrm{V}$ ratio ( $\mathrm{K}$, body urea clearance, $\mathrm{T}$, time of dialysis and $\mathrm{V}$, urea distribution volume) by machine derived online clearance monitoring (OCM) [3].

All patients were subjected to thorough history taking, clinical examination and follow up throughout the study period. All patients received recombinant human Erythropoietin (rhEPO). rhEPO doses were adjusted as needed to maintain $\mathrm{Hb}$ in the range of 11 to $12 \mathrm{~g} / \mathrm{dl}$.

All 16 patients were put on HF-HD for 3 months, then shifted to OL-HDF for 3 months. Patients were subjected to complete physical assessment including anthropometric and BP measurements. For the sake of comparison between patients, we divided the weight and height by the median weight and height for age and sex respectively. Laboratory tests included CBC, serum urea and creatinine by Beckman synchrony automated clinical system $\mathrm{Cx}$ 5, albumin, $\mathrm{Ca}, \mathrm{P}$, Parathyroid hormone (PTH), Na, K, alkaline phosphatase levels (ALP), KT/V and iron indices. All tests were done at the end of a period of 3 months of HF-HD and repeated 3 months after initiation of OL-HDF.

Nominal data were expressed as frequency and percentage. Numerical data were expressed as median and range for non-parametric data mean and standard deviation for parametric data. Comparisons had been done using chisquare tests for nominal and student t-tests for parametric data. Associations had been studied using Pearson's correlations. $\mathrm{P}$ values less than 0.05 were considered significant. Data were subjected to computer assisted statistical analysis using SPSS package version 16.0.

\section{Results}

Females represented $75 \%(\mathrm{~N}=12)$, with a female to male ratio $3: 1$. The mean age of the patients was $10.31 \pm 3.281$ yrs. (range 4 to 16 years). The mean weight of patients was $19.781 \pm 6.1724 \mathrm{~kg}$ (range 10.4-32.3 kg) and the mean height was $111.75 \pm 15.71 \mathrm{~cm}$ (range $80-135 \mathrm{~cm}$ ). Weight was $53.95 \pm 13.72 \%$ (range 27.5 to $76.6 \%$ ) and height was $79.54 \pm 6.21 \%$ of normal (range 64.9 to $87 \%$ ). Patients 'mean body surface area was $0.81 \pm 0.18 \mathrm{~m}^{2}$ (range 0.48 $1.11 \mathrm{~m}^{2}$ ). The mean body mass index was $18.01 \pm 1.89$ (range 15.2-22.2).

Congenital anomalies of the kidney and urinary tract (CAKUT) constituted the majority of causes of ESRD among our patients (7 patients, $43.9 \%$ ); $25 \%$ (4 patients) had end stage renal disease (ESRD) of undetermined etiology, other causes included chronic tubulointerstitial nephritis (2 patients, $12.5 \%$ ) and glomerulopathies $(6.3 \%$ of cases). There was no statistically significant difference in our study group as regards the dose of rhEPO requirements (mean $379.75 \pm 240.63 \mathrm{IU} / \mathrm{Kg} /$ week) on both dialysis modalities. (Table1) showing comparison between mean blood pressure values (in $\mathrm{mmHg}$ ) on high-flux (Table 2) showing different laboratory parameters OL- HDF and HF HD 
Table 1: Comparison between mean blood pressure values (in $\mathrm{mmHg}$ ) on high-flux HD \& online HDF.

\begin{tabular}{|c|c|c|c|c|c|c|}
\hline & & \multicolumn{2}{|c|}{ HDF } & \multicolumn{2}{|c|}{ High-flux HD } & \multirow{2}{*}{ p value ${ }^{*}$} \\
\hline & & Mean & SD & Mean & SD & \\
\hline \multirow{2}{*}{ SBP (mmHg) } & Pre & 124.69 & 15.86 & 118.75 & 14.09 & $\mathbf{0 . 0 3}^{*}$ \\
\hline & after 3 months & 114.50 & 10.98 & 111.44 & 12.36 & $0.01^{*}$ \\
\hline \multirow{2}{*}{ DBP(mmHg) } & Pre & 82.81 & 10.16 & 77.75 & 9.55 & $0.01 *$ \\
\hline & after 3 months & 72.19 & 6.57 & 77.13 & 8.37 & 0.32 \\
\hline \multirow[b]{2}{*}{ MBP(mmHg) } & Pre & 96.77 & 11.68 & 91.42 & 10.79 & 0.86 \\
\hline & after 3 months & 86.29 & 7.79 & 108.7 & 8.96 & 0.62 \\
\hline \multicolumn{7}{|c|}{ P- Values $<0.05$ are considered significant. } \\
\hline
\end{tabular}

Table 2: Different laboratory parameters OL- HDF and HF HD.

\begin{tabular}{|c|c|c|c|c|c|}
\hline & \multicolumn{2}{|c|}{ HDF } & \multicolumn{2}{|c|}{ High-flux HD } & \multirow{2}{*}{ P-Value* } \\
\hline & Mean & SD & Mean & SD & \\
\hline \multicolumn{6}{|c|}{ Anemia Parameters } \\
\hline Hb (g/dl) & 9.33 & 1.77 & 9.7 & 1.6 & 0.52 \\
\hline HCT $(\%)$ & 28.73 & 5.64 & 31.65 & 6.51 & 0.16 \\
\hline Ferritin (ng/ml) & 553 & 306.63 & 827.38 & 662.35 & 0.06 \\
\hline \multicolumn{6}{|c|}{ Bone function parameters } \\
\hline $\mathrm{Ca}(\mathrm{mg} / \mathrm{dl})$ & 8.83 & 1.03 & 7.92 & 0.87 & $\mathbf{0 . 0 2} *$ \\
\hline$P(\mathbf{m g} / \mathrm{dl})$ & 4.92 & 1.90 & 4.25 & 1.39 & $0.04 *$ \\
\hline CaxP & 45.19 & 19.13 & 33.21 & 10.54 & $\mathbf{0 . 0 0 3}^{*}$ \\
\hline $\operatorname{ALP}(\mathbf{I U} / \mathbf{L})$ & 836.44 & 788.66 & 705 & 962 & 0.21 \\
\hline PTH (pg/ml) & 749 & 788.66 & 404 & 476 & 0.001* \\
\hline \multicolumn{6}{|c|}{ Dialysis Adequacy parameters } \\
\hline Urea (mg/dl) & 78.56 & 31.63 & 64.4 & 14.325 & $\mathbf{0 . 0 2}^{*}$ \\
\hline Creatinine (mg/dl) & 9.819 & 4.87 & 8.38 & 3.79 & 0.21 \\
\hline KT/V & 2.08 & 0.47 & 1.75 & 0.46 & 0.006 \\
\hline \multicolumn{6}{|c|}{ Electrolytes } \\
\hline $\mathrm{Na}(\mathrm{mmol} / \mathrm{L})$ & 134.69 & 2.87 & 134 & 2.22 & 0.48 \\
\hline $\mathrm{K}(\mathrm{mmol} / \mathrm{L})$ & 4.98 & 1.26 & 4.58 & 0.81 & 0.15 \\
\hline \multicolumn{6}{|c|}{ Nutrition Parameters } \\
\hline Albumin (g/dl) & 3.52 & 0.34 & 3.35 & 0.48 & 0.22 \\
\hline
\end{tabular}




\section{Discussion}

Anemia is a major metabolic derangement in CKD, it is commonly hypo-proliferative, normochromic and normocytic and indistinguishable from anemia of chronic disease [4]. CKD patients have lower plasma levels of erythropoietin and less erythropoiesis than other patients with similar degree of anemia, suggesting inadequate production of erythropoietin by the diseased kidney as the primary mechanism of renal anemia [5]. Comparing HFHD to OLHDF regarding the control of anemia and the dosage of erythropoietin in our study group, showed no significant difference in the levels of $\mathrm{Hb}, \mathrm{HCT}$ and ferritin in both groups. No significant difference in the doses of rhEpo required to achieve adequate control of anemia (defined in our study as $\mathrm{Hb}$ in the range of 11 to $12 \mathrm{~g} / \mathrm{dl}$ ) in patients on HDF compared to high-flux HD was found either. Other studies comparing HDF with HD did not find a significant difference in terms of EPO responsiveness [6] or hemoglobin level [7]. Vaslaki et al. however found a higher Hct level at a comparable EPO dose in adult patients, who converted from HD to HDF [8].

Uremic syndrome results from malfunctioning of various organ systems due to retention of compounds, which under normal conditions, would be excreted into the urine and/or metabolized by the kidneys. If these compounds are biologically active, they are called uremic toxins [7]. Dialysis efficiency expressed by $\mathrm{Kt} / \mathrm{V}$ was significantly improved by HDF as compared to HF HD. Whether this is secondary to the higher convection volumes required for HDF alone is not clear. Many studies argue for an advantage of HDF over HF -HD in improving Kt/V [9, 10], on the other hand other studies show no difference between the two renal replacement modalities $[6,7]$.

There was a statistically insignificant reduction in post dialysis sNa during the OLHDF and the HFHD (134.69 +/2.869 and $134+/-2.221$, respectively, $\mathrm{P}=0.484)$. This agreed with Hwang et al who reported that pre and postdialysis sNa were not significantly different between OL HDF and HFHD [11]. On the other hand, Jean et al. found that compared to HF-HD, OL-HDF has a greater serum lowering effect for $\mathrm{Na}$, which might explain the ability of OL-HDF to stabilize both pre and post-dialysis SBP [12]. Although we could not reproduce the same results regarding serum $\mathrm{Na}$, we found statistically significant difference in SBP among our study group (114.50 \pm 10.9 on OL-HDF vs $111.4 \pm 12.3$ on HF-HD, $\mathrm{P}=0.013$ ) but not in DBP. In addition, increased removal of higher molecular weight uremic toxins like $\beta 2$-microglobulin, leptin and advanced glycation end products [AGE]) influence longterm dialysis-related vascular disease.

HDF has been reported to remove $\mathrm{P}$ more efficiently than HF-HD [8, 1], however our study showed statistically significant higher levels of $\mathrm{P}$ on HDF.

In the majority of studies comparing OL-HDF to HF$\mathrm{HD}, \mathrm{Ca}$ and PTH levels did not differ significantly, whereas our results showed higher levels of Ca and PTH on HDF $[6,10]$. Similar studies on adult patients show contradicting results. Rius et al. showed that PTH levels are reduced after a session with low volume HDF, but increase after high volume HDF, possibly secondary to negative calcium balance [13]. Jean et al did not observe any differences in $\mathrm{P}$ levels in HDF compared to HF-HD patients [14], while Lornoy et al. reported $\mathrm{P}$ mass removal to be enhanced by $15-20 \%$ after shifting to HDF [15] also Francisco et al reported lower P level at the end of his study in HDF patients compared to HFHD (3.4=/-0.8 versus 4.5 +/- $1.6 \mathrm{mg} / \mathrm{dl}, \mathrm{P}<0.05)$ [16]. In one large study by Penne et al, predialysis $P$ levels in HDF were reduced by $6 \%$ and the percentage of patients reaching phosphate treatment targets increased from $64 \%$ to $74 \%$ after 6 months of HDF, however this is not a uniform finding, and may depend on membrane characteristics [17]. Movilli et al. reported significant reduction of serum $\mathrm{P}$ (from $5.1+/-1.0$ to $4.0+/$ $0.7, \mathrm{P}<0.0001$ ) and PTH concentrations (from $307+/-167$ to $194+/-98, \mathrm{P}<.0001)$ while there was no significant change $\mathrm{Ca}$ and phosphate binder dose on post-dilutional HDF [10].

It is not possible to make a significant conclusion from our assessment of bone parameters in both modalities, since the duration of both treatment modalities was short (3 months) compared to the previously mentioned studies. We found no studies on comparable age groups to guide the judgment of our results, and no data to estimate the duration on each modality needed to affect the different studied parameters.

We found no significant difference in albumin levels in HDF patients compared to HD patients $3.519+/-0.3391$ versus $3.350+/-0.48+/-0.219(\mathrm{P}=0.219)$. Wizemann et al, reported no significant difference in HDF compared to HD patients [19]. As regard the nutritional marker in this study represented by the albumin if we considered the large dialyzer surface area, the membrane porosity and the high amount of convective transport in HDF, an increased loss of valuable amino acids, peptides and proteins, e.g. albumin can be expected. On the other side removal of certain middle molecules like leptin improves appetite and consequently malnutrition. We found that there was no difference in plasma albumin between both study groups. Also Locatelli et al did not demonstrate an influence of dialysis membrane or convection on any of the variables related to the nutritional status (body weight, serum albumin etc.) [20]. Several other studies did not prove a significant difference between serum albumin levels in patients treated with HD versus patients treated with HDF $[6,7]$.

Albumin level reduction with HDF was reported by several authors [21, 22], but without having a clear clinical impact. Combarnous et al. studied albumin removal with predilutional HDF, most likely due to an interaction with the polymer surface of the dialysis membrane. Albumin loss during HDF seemed to have no acute impact on plasma albumin [23].

Our study has several limitations: First, the design of our study was not a randomized, controlled study but rather was a analytical non-randomized study. Limitations also include the small size of the study group and the length of the study. Reasons behind this is the high turnover of patients in our unit, as most children are prepared for transplantation as soon as possible, and the economic burden of maintaining children on HDF. We recommend further studying the comparison of both modalities over longer periods, and stratification of patients according to comorbidities, e.g. hypertension. 


\section{Conclusions}

We found no advantages of HDF over high-flux HD with respect to anemia management, metabolic bone disease and nutrition, however HDF proved to be more beneficial in dialysis efficiency and control of SBP.

\section{References}

1. Canaud B, Bowry S and Stuard s: Hemodiafiltration: in Handbook of Dialysis (5th Edition).

Lippincot \& Williams, 2015, p.321.

2 DiRaimondo CR, Pollak VE Beta 2-microglobulin kinetics in maintenance hemodialysis: a comparison of conventional and high-flux dialyzers and the effects of dialyzer reuse. Am J Kidney Dis (1989), 13: 390-395

3. Daugirdas JT. Second generation logarithmic estimates of single-pool variable volume $\mathrm{Kt} / \mathrm{V}$ : an analysis of error. J Am Soc Nephrol 1993; 4(5) 1205-13.

4. Weiss G,Goodnough LT Anemia of Chronic Disease. N Eng J Med; 2005, 352:1011-1023.

5. Fischbach M, Attal Y Geisert J: Hemodiafiltratio versus Hemodialysis in children,

Int J Pediat Nephrol, 1984, 5: 151-154.

6. Vilar E, Fry AC, Wellsted D, Tattersall JE, Greenwood $\mathrm{RN}$, Farrington $\mathrm{K}$ Long-term outcomes in online hemodiafiltration and high-flux hemodialysis: a comparative analysis.

Clin J Am Soc Nephrol, 2009, 4(12):1944-1953

7. Oates T, Pinney JH, Davenport A Haemodiafiltration versus high-flux haemodialysis: effects on phosphate control and erythropoietin response.

Am J Nephrol, 2011, 33(1):70-75.

8. Vaslaki L, Major L, Berta K, Karatson A, Misz M, Pethoe F, Ladanyi E, Fodor B, Stein G, Pischetsrieder M, Zima T, Wojke R, Gauly A, Passlick-Deetjen J On-line haemodiafiltration versus haemodialysis: stable haematocrit with less erythropoietin and improvement of other relevant blood parameters.

Blood Purif, 2006, 24:163-173

9. Schiffl H Prospective randomized cross-over longterm comparison of online haemodiafiltration and ultrapure high-flux haemodialysis. Eur J Med Res 2007, 12(1):26-33 10. Movelli E Camerini et al: Effect of post dilutional online Haemodiafiltration on serum calcium,phosphate and parathyroid hormone concentrations in uremic patients. Nephrology Dialysis Transplantation 2011, vol 25 Issue 12 p 4032-4037.

11. Hwang KS, Choi EY et al: Post dialysis serum sodium changes and systolic blood pressure in patients undergoing.

\section{Declaration}

\section{Ethics approval and consent to participate}

This study protocol and the consents were approved and deemed sufficient by Ethical Committee of Pediatric Department, Faculty of Medicine, Cairo University. And informed written consent was obtained in every case from their legal guardians.

Funding: The authors declare that they didn't receive any financial support from agencies or others.

Conflict of interest: $\mathrm{No}$

\section{Acknowledgements}

We would like to thank all patients and their family members for their valuable contributions to the study.
On-line hemodiafiltration and high flux hemodialysis. Kidney Res Clin Pract: 2013, 32(2): 62-65.

12. Ward RA, Schmidt B, Hullin J, Hillebrand GF, Samtleben W a comparison of on-line hemodiafiltration and high-flux hemodialysis: a prospective clinical study. J Am Soc Nephrol 2000, 11(12):2344-2350

13. Rius A, Hernandez-Jaras J, Pons R, Garcia PH, Torregrosa E, Sanchez Canel JJ, et al. Kinetic of calcium, phosphate, magnesium and PTH variations during hemodiafiltration. Nefrología. 2007; 27(5):593-8

14. Jean G, Hurot J-M et al: On-line Haemodiafiltration Vs Conventional Haemodialysis: A cross-over study. BMC Nephrol; 2015, 16-70. Published online May 9

15. Lornoy $\mathrm{W}$ et al: Impact of convective flow on phosphorus removal in maintenance Haemodialysis patients, J Ren Nut, 2006, 16: 47-53.

16. Francisco RC, Aloha M and Ramon PS: Effects of high-efficiency post diafiltration and high flux hemodialysis on serum phosphorus and cardiac structure and function in patients with end-stage renal disease. International Urology and Nephrology. 2013, vol 45 Issue 5 p 1373-1378.

17. Penne EL, van der Weerd NC, van der Dorpel MA: Short-term Effects of Online Hemodiafiltration on Phosphate Control: A Result from the Randomized Controlled Convective Transport Study (CONTRAST). Am J Kid Dis. 2010, 55 (1): 77-87

18. Zehnder C, Gutzwiller JP, Renggli K Hemodiafiltration - a new treatment option for hyperphosphatemia in hemodialysis patients. Clin Nephrol, 1999, 52(3):152-159

19. Wizemann V, Latz C, Techert F and Uthoff: On-line haemodiafiltration versus low-flux haemodialysis. A prospective randomized study .Nehrology Dialysis transplantation, 2000, vol 15, Issue suppl -1, p 43-48.

20. Locatelli F, Altieri P, Andrulli S, Bolasco P, Sau G, PedriniLA,Basile C,David S,Feriani M,Montagna G,DiIorio BR, Memoli B, Cravero R, Battaglia G, Zoccali $\mathrm{C}$ Hemofiltrati on and hemodiafiltration reduce intradialytic hypotension in ESRD.

J Am Soc Nephrol, 2010, 21(10): 1798-1807

21. Stefánsson BV, Abramson M, Nilsson U, Haraldsson B Hemodiafiltration improves plasma 25-hepcidin levels: a prospective, randomized, blinded, cross-over study comparing hemodialysis and hemodiafiltration. Nephron Extra 2012, 2(1):55-65

22. Tomo T, Matsuyama K, Nasu M Effect of hemodiafiltration against radical stress in the course of blood purification. Blood Purif (2004) 22(suppl 2):72-77 23. Combarnous F, Tetta C, Cellier CC, Wratten ML, Custaud MA, De Catheu T, Fouque D, David S, Carraro G, Laville M Albumin loss in on-line hemodiafiltration.

Int J Artif Organs 2. 2002,5(3):203-209. 